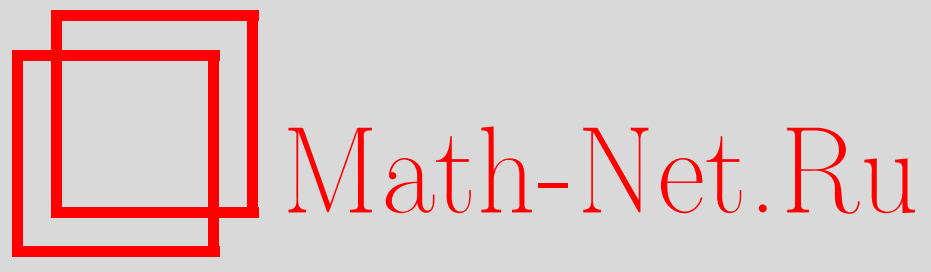

В. В. Дорошенко, Большинство полугрупп преобразований свободны, Матем. заметки, 2010, том 87, выпуск $3,464-467$

DOI: https://doi.org/10.4213/mzm8676

Использование Общероссийского математического портала Math-Net.Ru подразумевает, что вы прочитали и согласны с пользовательским соглашением http://www . mathnet.ru/rus/agreement

Параметры загрузки:

IP : 54.162 .85 .209

26 апреля 2023 г., 09:48:15

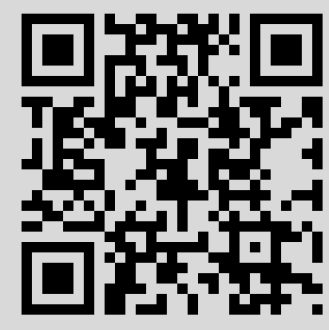




\section{Большинство полугрупп преобразований свободны}

\section{В. В. Дорошенко}

1. Введение. Пусть $\mathbb{N}$ - множество всех натуральных чисел. Множество $T(\mathbb{N})$ всех преобразований $\mathbb{N}$ относительно композиции $f g=f(g), f, g \in T(\mathbb{N})$, образует полугруппу, которую называют полной полугруппой преобразований натурального рлда. В данной работе показано, что в самой полугруппе $T(\mathbb{N})$ и в континуальном классе ее подполугрупп в некотором смысле большинство конечно порожденных подполугрупп являются свободными. Аналогичные результаты о свободных подгруппах симметрической группы счетной степени получены в [1], а о свободных подполугруппах полугруппы эндоморфизмов корневого дерева и некоторых матричных полугрупп в [2] и [3] соответственно.

Пусть $(X, \rho)$ - метрическое пространство. Для $\varepsilon>0$ и $x \in X$ определим $\varepsilon$-шар

$$
B(f, \varepsilon)=\{y \in X \mid \rho(x, y)<\varepsilon\} .
$$

Для натурального $N$ и $x \in X$ обозначим $B_{N}(x)=B\left(x, 2^{-N}\right)$. Для каждого $m \in \mathbb{N}$ будем рассматривать $m$-кратную декартову степень $\left(X^{m}, \rho_{m}\right)$, где $X^{m}$ - декартова степень множества $X$, а для $x=\left(x_{1}, \ldots, x_{m}\right), y=\left(y_{1}, \ldots, y_{m}\right) \in X$ имеем

$$
\rho_{m}(x, y)=\max _{1 \leqslant k \leqslant m} \rho\left(x_{k}, y_{k}\right) .
$$

Пусть $X$ является полугруппой, в которой умножение непрерывно относительно метрики $\rho$, т.е. $X$ - непрерывная полугруппа. Свободной полугруппой $F_{m}$ ранга $m$ со свободными порождающими $x_{1}, \ldots, x_{m}$ является множество всех слов над алфавитом $x_{1}, \ldots, x_{m}$ относительно операции приписывания слов. Значением слова $w=x_{n_{1}} \ldots x_{n_{k}} \in F_{m}$ от элементов $a_{1}, \ldots, a_{m} \in X$ будем называть произведение $a_{n_{1}} \cdots a_{n_{k}}$, которое обозначим $w\left(a_{1}, \ldots, a_{m}\right)$. Будем говорить, что полугруппа $X$ обладает свойством типичности (ubiquity) свободных подполугрупп, если для любого $m \in \mathbb{N}$ множество всех таких наборов $\left(x_{1}, \ldots, x_{m}\right) \in X^{m}$, что полугруппа $\left\langle x_{1}, \ldots, x_{m}\right\rangle$, порожденная компонентами каждого из них, изоморфна свободной полугруппе $F_{m}$, является множеством второй категории в $\left(X^{m}, \rho_{m}\right)$, а его дополнение - множеством первой категории.

Введем метрику на $T(\mathbb{N})$. Для $f, g \in T(\mathbb{N})$ положим

$$
\rho(f, g)= \begin{cases}0, & f=g, \\ 2^{-n}, & f(k)=g(k), k=1, \ldots, n-1, f(n) \neq g(n) .\end{cases}
$$

Легко видеть, что $\rho$ действительно есть метрика. Заметим, что для $f \in T(\mathbb{N})$ и $N \in \mathbb{N}$ шар $B_{N}(f)$ состоит из всех функций, совпадающих с $f$ на множестве $\{1, \ldots, N\}$.

Заметим, что любой $\varepsilon$-шар равен некоторому $2^{-N}$-шару. Поэтому достаточно рассматривать только $2^{-N}$-шары.

ПреДЛОЖЕНИЕ 1.1. Умножение в $T(\mathbb{N})$ непрерывно относителъно $\rho$.

ПРеДЛОЖение 1.2. $(T(\mathbb{N}), \rho)$ - полное метрическое пространство.

2. Основная теорема. Пусть $S$ - подполугруппа $T(\mathbb{N})$. Тогда $S$ будет метрическим пространством с метрикой, индуцированной из $T(\mathbb{N})$. Зафиксируем натуральное число $m$.

Определим множество

$$
C_{m}=\left\{\left(f_{1}, \ldots, f_{m}\right) \in S^{m} \mid\left\langle f_{1}, \ldots, f_{m}\right\rangle \text { изоморфна } F_{m}\right\} .
$$

(C) В. В. Дорошенко, 2010 
Для произвольных $w_{1}, w_{2} \in F_{m}, w_{1} \neq w_{2}$, положим

$$
A_{w_{1} w_{2}}=\left\{\left(f_{1}, \ldots, f_{m}\right) \in S^{m} \mid w_{1}\left(f_{1}, \ldots, f_{m}\right)=w_{2}\left(f_{1}, \ldots, f_{m}\right)\right\} .
$$

Тогда очевидно равенство $C_{m}=S^{m} \backslash \bigcup A_{w_{1} w_{2}}$, где объединение берется по всем парам различных слов из свободной полугруппы $F_{m}$.

Доказательство следующей леммы стандартно.

Лемма 2.1. Для произвольных $w_{1}, w_{2} \in F_{m}, w_{1} \neq w_{2}$, множество $A_{w_{1} w_{2}}$ замкнуто в метрическом пространстве $\left(S^{m}, \rho_{m}\right)$.

Назовем отображение $f \in T(\mathbb{N})$ почти инъективным, если его сужение на некоторое подмножество с конечным дополнением в множестве $\mathbb{N}$ инъективно. Это означает, что число пар $(x, y)$ различных натуральных чисел таких, что $f(x)=f(y)$, конечно, т.е. существует натуральное $N$ такое, что для любых натуральных $x, y, x>y \geqslant N$ выполняется неравенство $f(x) \neq f(y)$.

Пусть $A \subset \mathbb{N}$. Будем говорить, что образ $f$ почти содержится в $A$, если множество

$$
\{x \in \mathbb{N} \mid f(x) \notin A\}=f^{-1}(\mathbb{N} \backslash A)
$$

конечно.

Лемма 2.2. 1) Произведение почти ингекиий есть почти ингекиия.

2) Пусть $f, g, h \in T(\mathbb{N})$ - почти ингекиии и множество $\{x \in \mathbb{N} \mid g(x) \neq h(x)\}$ бесконечно. Тогда $f g \neq f h$.

3) Пусть $f \in T(\mathbb{N})$ почти инвекиия, $A \subset \mathbb{N}, g \in T(\mathbb{N})$ и образ $g$ почти содержится в A. Тогда образ $g f$ почти содержится в $A$.

Доказательство. 1) Пусть $f, g \in T(\mathbb{N})$ почти инъекции. Обозначим символом $D$ множество всех натуральных чисел, прообраз каждого из которых относительно $f$ содержит более одного элемента. Тогда $D$ конечно. Рассмотрим множество

$$
C=\left\{(x, y) \in \mathbb{N}^{2} \mid x \neq y, f g(x)=f g(y)\right\} .
$$

Тогда

$$
\begin{aligned}
& C=\left\{(x, y) \in \mathbb{N}^{2} \mid x \neq y, g(x)=g(y)\right\} \\
& \cup\left\{(x, y) \in \mathbb{N}^{2} \mid x \neq y, g(x) \neq g(y), f(g(x))=f(g(y))\right\} .
\end{aligned}
$$

Первое множество в объединении конечно по определению почти инъекции, а второе содержится в

$$
\left\{(x, y) \in \mathbb{N}^{2} \mid g(x), g(y) \in D\right\}=g^{-1}(D) \times g^{-1}(D)
$$

и поэтому конечно, так как прообраз конечного множества относительно почти инъективного отображения конечен. Итак, множество $C$ конечно и поэтому $f g$ - почти инъекция.

2) Существует натуральное $N$ такое, что для всех $x, y \in \mathbb{N}, x>y \geqslant N$, имеет место неравенство $f(x) \neq f(y)$. Выберем натуральное $M$ так, что для всех $x \in \mathbb{N}, x \geqslant M$, выполнены неравенства $g(x), h(x) \geqslant N$. Поскольку $g, h$ отличаются в бесконечном количестве точек, существует натуральное $x \geqslant M$ такое, что $g(x) \neq h(x)$. Тогда $f g(x) \neq f h(x)$.

3) Рассмотрим множество $(g f)^{-1}(\mathbb{N} \backslash A)=f^{-1}\left(g^{-1}(\mathbb{N} \backslash A)\right)$. Множество $g^{-1}(\mathbb{N} \backslash A)$ конечно, так как образ $g$ почти содержится в $A$, а прообраз конечного множества относительно почти инъективного отображения конечен. Поэтому множество $(g f)^{-1}(\mathbb{N} \backslash A)$ конечно и получаем требуемое.

Определим следующие условия для полугруппы $S$.

УСловие 1. Полугруппа $S$ - замкнутое подмножество в метрическом пространстве $(T(\mathbb{N}), \rho)$. 
Условие 2. Существуют бесконечные непересекающиеся множества $A, B \subset \mathbb{N}$ такие, что для любого отображения $f \in S$ в любой окрестности $f$ найдутся почти инъективные $g, h \in S$, для которых образ $g$ почти содержится в $A$ и образ $h$ почти содержится в $B$.

Лемма 2.3. Если полугруппа $S$ удовлетворяет условию 2, то для любых различных $w_{1}, w_{2} \in F^{m}$ множество $A_{w_{1} w_{2}}$ не содержит внутренних точек.

Доказательство. Пусть $f=\left(f_{1}, \ldots, f_{m}\right) \in A_{w_{1} w_{2}}$. Докажем, что $f$ - не внутренняя точка $A_{w_{1} w_{2}}$. Зафиксируем $N>0$. Пусть $w_{1}=u v_{1}, w_{2}=u v_{2}$, где $u$ - общий префикс, а слова $v_{1}, v_{2}$ начинаются с разных букв или одно из них пусто. По условию 2 существуют почти инъекции $g_{i}, h_{i} \in B_{N}\left(f_{i}\right), 1 \leqslant i \leqslant m$, такие, что образы $g_{i}, h_{i}$ почти попадают в $A$ и $B$ соответственно. Пусть

$$
l_{i}=\left\{\begin{array}{ll}
g_{i}, & \text { если } x_{i} \text { не первая буква } v_{2}, \\
h_{i}, & \text { если } x_{i} \text { первая буква } v_{2},
\end{array} \quad 1 \leqslant i \leqslant m .\right.
$$

Тогда $l=\left(l_{1}, \ldots, l_{n}\right) \in B_{N}(f)$. Докажем, что $v_{1}(l) \neq v_{2}(l)$. Есть две возможности.

1) Одно из слов $v_{1}, v_{2}$ пусто. Пусть пусто $v_{1}$. По лемме $\left.\left.2.2,1\right), 3\right)$ образ $v_{2}(l)$ почти содержится в $B$, т.е. является собственным подмножеством $\mathbb{N}$. Образ $v_{1}(l)$ равен $\mathbb{N}$ и поэтому $v_{1}(l) \neq v_{2}(l)$.

2) Оба слова $v_{1}, v_{2}$ не пусты и начинаются с различных букв. По лемме $\left.2.2,1\right), 3$ ) образ $v_{1}(l)$ почти содержится в $A$, образ $v_{2}(l)$ почти содержится в $B$. Поскольку $A \cap B=\varnothing$, то $v_{1}(l)$ и $v_{2}(l)$ не совпадают на бесконечном множестве точек.

По лемме $2.2,2) \quad w_{1}(l) \neq w_{2}(l)$; следовательно, $l \notin A_{w_{1} w_{2}}$.

Сформулируем теперь основную теорему.

Теорема 2.1. Если $S$ удовлетворяет условиям 1 и 2, то $C_{m}$ есть множество второй категории, а его дополнение - первой.

ДоказАтельство. Для любых различных $w_{1}, w_{2} \in F_{m}$ по леммам 2.1 и 2.3 множество $A_{w_{1} w_{2}}$ замкнуто и не содержит внутренних точек. Поэтому оно нигде не плотно в $S^{m}$. Тогда

$$
\bigcup_{w_{1}, w_{2} \in F_{m}, w_{1} \neq w_{2}} A_{w_{1} w_{2}}
$$

- множество первой категории. По условию 1 пространство $(S, \rho)$ полно, поскольку $(T(\mathbb{N}), \rho)$ полно. По теореме Бэра о категории [4] $\left(S^{m}, \rho_{m}\right)$ есть множество второй категории, поэтому $C_{m}=S^{m} \backslash \bigcup_{w_{1} \neq w_{2}} A_{w_{1} w_{2}}$ - множество второй категории, а его дополнение первой.

3. Примеры. 1. Рассмотрим вначале саму полугруппу $T(\mathbb{N})$. Для нее оказывается справедливой

Теорема 3.1. Полугруппа $T(\mathbb{N})$ обладает свойством типичности свободных подполугрупп.

2. Обозначим символом $O(\mathbb{N})$ подполугруппу $T(\mathbb{N})$, состоящую из всех возрастающих функций из $\mathbb{N}$ в $\mathbb{N}$, т.е. $f \in T(\mathbb{N})$ принадлежит $O(\mathbb{N})$, если $f(n)<f(n+1)$ для всех $n \in \mathbb{N}$. Рассмотрим метрическое пространство $(O(\mathbb{N}), \rho)$ с метрикой, индуцированной из $T(\mathbb{N})$. Умножение в $O(\mathbb{N})$ также непрерывно относительно $\rho$.

ПреДЛОЖеНИЕ 3.1. Полугруппа $O(\mathbb{N})$ - замкнутое подмножество в $T(\mathbb{N})$.

ДоказАтельство. Нужно показать, что в $(T(\mathbb{N}), \rho)$ предел функций из $O(\mathbb{N})$ снова принадлежит $O(\mathbb{N})$. Это следует из того, что если $f_{n} \rightarrow f, n \rightarrow \infty$ в $(T(\mathbb{N}), \rho)$, то для всех $m \in \mathbb{N}$, начиная с некоторого номера, все функции $f_{n}$ принимают одинаковые значения на $m$. 
ПреДЛОЖЕНИЕ 3.2. Полугруппа $O(\mathbb{N})$ обладает свойством типичности свободных подполугрупп.

Аналогично показывается, что подполугруппа всех неубывающих функций из $\mathbb{N}$ в $\mathbb{N}$ и полугруппа всех инъективных преобразований $\mathbb{N}$ обладают свойством типичности свободных подполугрупп.

3. Пусть $X-$ бесконечное подмножество $\mathbb{N}$. Обозначим

$$
T_{X}=\{f \in T(\mathbb{N}) \mid f(\mathbb{N}) \subset X\} .
$$

Легко видеть, что $T_{X}$ - замкнутая подполугруппа $T(\mathbb{N})$.

ПРЕДЛОЖенИЕ 3.3. Полугруппа $T_{X}$ обладает свойством типичности свободных подполугрупп.

СледСтвиЕ 1. Полугруппа $T(\mathbb{N})$ содержит континуальное семейство подполугрупn, каждая из которых обладает свойством типичности свободных подполугрупп.

4. Во всех предыдущих примерах утверждалось, что свободных подполугрупп много, но не было приведено ни одного примера свободной подполугруппы. Предъявим явный пример свободной подполугруппы в $T(\mathbb{N})$.

Определим $f_{0}, f_{1} \in T(\mathbb{N})$ следующим образом:

$$
f_{0}(n)=2 n, \quad f_{1}(n)=2 n+1, \quad n \in \mathbb{N} .
$$

Тогда верна следующая теорема.

Теорема 3.2. Функции $f_{0}, f_{1}$ порождают свободную подполугруппу ранга 2 в $O(\mathbb{N})$.

Аналогично показывается, что преобразования $g_{0}, \ldots, g_{m-1}$, заданные формулами

$$
g_{i}(n)=m n+i, \quad 0 \leqslant i<n,
$$

порождают свободную подполугруппу $O(\mathbb{N})$ ранга $m$.

Отметим, что результаты этой статьи можно перенести на полугруппу $P T(\mathbb{N})$ всех частично определенных преобразований множества $\mathbb{N}$.

\section{СПИСОК ЦИТИРОВАННОЙ ЛИТЕРАТУРЫ}

[1] J. D. Dixon, Bull. London Math. Soc., 22:3 (1990), 222-226. [2] А. С. Олийнык, Матем. заметки, 63:2 (1998), 248-259. [3] W. Hołubowski, Semigroup Forum, 66:2 (2003), 231-235. [4] Р. А. Александрян, Э. А. Мирзаханян, Общая топология, Высшая школа, M., 1979.

\section{В. В. Дорошенко}

Киевский национальный университет им. Т. Г. Шевченко, Украина

E-mail: dvadim@univ.kiev.ua
Поступило

31.01.2006

Исправленный вариант

03.06.2009 